\title{
Brandstetter, Bettina (2020). Kulturen, Religionen und Identitäten aushandeln. Elementarpädagogik zwischen Homogenisierung und Pluralisierung (Interreligiöse und interkulturelle Bildung im Kindesalter 9). Münster: Waxmann. ISBN 978-3-8309-4I39-2. 268 Seiten.
}

\author{
Helena Stockinger \\ Ludwig-Maximilians-Universität München (helena.stockinger@lmu.de)
}

Die überarbeitete Dissertation von Bettina Brandstetter geht den „Konfrontationen und Komplexitäten nach, die im Kindergarten vorranging im Zusammenhang mit kultureller Diversität und religiöser Pluralität auftreten“ (14f.) und zeigt diese anhand von Interviews mit Elementarpädagog*innen auf. Das Buch möchte gesellschaftliche Strukturen und Machtverhältnisse offenlegen, um diese einer theologischen Bearbeitung zuzuführen, woraus sowohl Lernimpulse für die Theologie als auch für elementarpädagogische Bildungsräume erwachsen sollen. Die Pädagog*innen als Subjekte rücken in den Hintergrund, da die „Wechselwirkungen zwischen gesellschaftlichen Diskursen, Repräsentationen und Ungleichheitsverhältnissen“ (21) im Bildungsraum Kindergarten erforscht werden möchten. Die Arbeit, die im Fach Theologie interkulturell verortet ist, umkreist in fünf Teilen die Auseinandersetzung mit Homogenisierungs- und Pluralisierungsdynamiken.

Nach einer Einleitung, in der das Grundverständnis des Kindergartens offen gelegt wird, erfolgt eine theoretische Einbettung der Arbeit, die sich an der Diskurstheorie nach Michel Foucault, der Diskursanalyse (Siegfried Jäger) und der Tradition der Postkolonialen Theorien (Edward W. Said, Gayatri Chakravorty Spivak, Homi K. Bhabha) orientiert, um davon ausgehend „das diskursive Netz, in das Homogenisierungs- und Pluralisierungsstrategien eingewoben sind“ (25) unter Berücksichtigung von Ausarbeitungen von Safiye Yildiz zu skizzieren. Im Mittelpunkt der anschließenden qualitativ-empirischen Untersuchung stehen Interviews mit sechs Elementarpädagog*innen, in denen diese von ihren Wahrnehmungen und Erfahrungen mit kultureller Diversität und religiöser Pluralität im Kindergartenalltag erzählen. Forschungsmethodisch wird eine Forschungshaltung der Grounded Theory gepaart mit der Methodik der Inhaltsanalyse nach Mayring angestrebt, die anschließend einer postkolonial informierten Diskursanalyse unterzogen wird. In der Ergebnisdarstellung werden die Interviews der Pädagog*innen nacheinander beschrieben und einzelne Aussagen daraufhin befragt, ob diese Pluralisierungs- und Homogenisierungstendenzen folgen. Diejenigen Situationen, die nicht eindeutig zuordenbar sind, werden als Zwischenräume, in denen sich alternative Ordnungen erkennen lassen, ausführlich dargestellt. In diesen Aushandlungsräumen von Diskursen und Identitäten wird das kreative Handlungspotential von Kindern, Eltern und Pädagog*innen sichtbar. Die beiden anschließenden Teile entfalten die Wechselbeziehungen der Interkulturellen Theologie und des Bildungsortes Kindergarten. Beide Orte sind von der aktuellen gesellschaftlichen Situation angefragt und suchen nach Lösungen und Antworten, „die für eine gegenwärtige Identitätsfindung und für die kulturellen Ausverhandlungen solcher Identitätsfindungen wichtig und geradezu unumgänglich sind“ (189). Einerseits werden ausgehend von den empirischen Daten Fundstellen für die interkulturelle Theologie benannt und Ansprüche an diese formuliert. Es werden Fragen nach einem verantwortlichen Umgang mit Pluralität und angemessenen religionspädagogischen Entwürfen gestellt. Andererseits werden ausgehend von ausgewählten Salzburger religionstheologischen Entwürfen (Franz Gmainer-Pranzl, Sigrid Rettenbacher, Judith Gruber, Ulrich Winkler) diese interkulturellen Theologien daraufhin befragt, inwiefern sich die aufgeworfenen Problemlagen im Kindergarten widerspiegeln. In dieser Auseinandersetzung wird der Kindergarten als locus theologicus alienus bestimmt. Abschließend werden Anfragen an eine pluralitätssensible Religionspädagogik gestellt, indem zwei exemplarische Ansätze (Stephan Leimgruber, Friedrich Schweitzer) auf ihre Pluralisierungs- und Homogenisierungstendenzen befragt werden. Notwendige Kompetenzen der Elementarpädagog*innen werden benannt, wobei die aus dem empirischen Material abgeleitete Kompetenz des Zwischenraums näher entfaltet wird.

Bettina Brandstetter verortet ihre Auseinandersetzungen in der Tradition der postkolonialen Theorien und verfolgt konsequent die von ihr gewählte Perspektive. Die Verschränkung dieses Zugangs mit empirischen Erkenntnissen der Elementarpädagogik eröffnet sowohl Einsichten in den Bildungsort Kindergarten als auch Denkanstöße für die 
theoretische Auseinandersetzung mit Pluralität. Macht- und Herrschaftsverhältnisse sowie Identitätskonstruktionen, die auf einer zugeschriebenen Kultur oder Religion beruhen, werden hierdurch besonders deutlich. Die im Vorfeld getroffenen starken Vorannahmen, mit denen an die empirischen Daten herangegangen wird, werden transparent aufgezeigt. In der Ergebnisdarstellung geben die ausführlichen Beschreibungen der Interviews einen ungeschminkten Einblick in den Kindergartenalltag und verdeutlichen die Herausforderungen und Schwierigkeiten, mit denen Elementarpädagog*innen am Bildungsort Kindergarten regelmäßig konfrontiert sind. Die Sichtweisen und Handlungsstrategien der Pädagog*innen werden umfassend dargelegt und die einzelnen Aussagen großteils klar nachvollziehbar der Homogenisierungs- oder der Pluralisierungstendenz zugewiesen. Dabei eröffnen sich vielfältige interessante Einblicke in die Kindergartenpraxis und darin eingeschriebene Homogenisierungs- und Pluralisierungspraktiken, die durch zahlreiche - in einem österreichischen Dialekt - angeführte Originalzitate veranschaulicht werden. Die Erzählungen möchten nicht die Arbeit der Pädagog*innen im Feld abwerten, sondern die gesellschaftlichen Ordnungen und eingeschriebenen post-kolonialen und hegemonialen Verhältnisse aufzeigen. Dieser Zielsetzung gilt es sich beim Lesen des Buches bewusst zu bleiben, insbesondere wenn die auf Pluralisierung und Homogenisierung hin interpretierten Situationen Lesende etwas ratlos zurücklassen, wie mit bestimmten Situationen umgegangen werden kann, ohne in eine Pluralisierungs- oder Homogenisierungstendenz eingeordnet zu werden. Aufschlussreich sind hier die geschilderten Zwischenräume, die von Brandstetter in einzelnen Handlungen von Pädagog*innen identifiziert und nicht den beiden Tendenzen zugeordnet werden, sondern sich im Pendeln zwischen diesen einstellen.

Die Arbeit zeigt, wie gewinnbringend es sein kann, Erkenntnisse der interkulturellen Theologien und des Bildungsorts Kindergarten miteinander zu verschränken. Der Kindergarten wird als Ort deutlich, in dem sich die Problemlagen, die in den interkulturellen Theologien thematisiert werden, widerspiegeln und der gleichzeitig neue Fragen und Ansprüche aufwirft. Wenn die Arbeit auch in den interkulturellen Theologien verortet ist, bietet sie doch weiterführende Überlegungen für eine diskurssensible Religionspädagogik. Die Bedeutung, postkoloniale Theorien stärker in den religionspädagogischen Diskurs einzuspielen und ein Bewusstsein für in der pädagogischen Arbeit inhärente Machtdiskurse zu entwickeln, wird durch die Arbeit besonders deutlich. Für die Religionspädagogik ergiebig könnte es noch sein, die Erkenntnisse vertieft auf religionspädagogische Implikationen weiterzudenken und noch stärker in den Diskurs um eine pluralitätssensible Religionspädagogik einzubetten. Es ergeben sich hierfür vielfältige Anknüpfungspunkte, denen es weiter nachzugehen lohnt.

In der Studie von Bettina Brandstetter wird eindrucksvoll deutlich, dass der Forschung in der frühen Kindheit eine erkenntnisgenerierende Bedeutung für die Theologie zukommt und Erkenntnisse des wichtigen Bildungsortes Kindergarten auch andere Orte sowie die Religionspädagogik anfragen und herausfordern. 